\title{
Os preços dos medicamentos de referência após a entrada dos medicamentos genéricos no mercado farmacêutico brasileiro
}

\author{
Marislei Nishijima*
}

Sumário: 1. Introdução; 2. Revisão Bibliográfica; 3. Dados e Estatísticas Preliminares; 4. Preços dos Medicamentos de Referência: Modelo de Diferença em Diferenças; 5. Comportamento dos Preços dos medicamentos de marca conforme o número de medicamentos genéricos que entram no mercado; 6 . Conclusões.

Palavras-chave: medicamentos genéricos, preços, indústria farmacêutica, microdados.

Códigos JEL: L65, I11

Este artigo analisa o comportamento dos preços dos medicamentos pioneiros de marca que se tornaram referência de genéricos no mercado brasileiro. A análise é baseada em microdados provenientes de três fontes distintas. As estimativas foram obtidas usando modelos de diferenças em diferenças e de efeitos fixos. Os resultados sugerem que os preços dos medicamentos de referência responderam negativamente à entrada de genéricos em seus respectivos mercados no curto período de tempo estudado. Também sugerem que os preços dos medicamentos de referência são sensíveis ao número de genéricos existentes em seus mercados.

This paper analyses how prices of the original brand drugs are affected by generic entry in Brazilian pharmaceutical market. Microdata from three different sources are used. To estimate the parameters we used difference in difference and fixed effect models. The results suggest that the original brand prices decreased after generic entry. Also, the results suggest that these prices are sensible to the number of generic drugs in their markets.

\section{INTRODUÇÃO}

Uma tendência recente verificada em vários países é a de aumento relativo dos gastos com medicamentos frente aos gastos com demais bens (Lichtenberg, 2001). No Brasil, os gastos das famílias com

*Universidade de São Paulo E-mail: marislei@usp.br Av. Arlindo Béttio, 1000, Ermelino Matarazzo, São Paulo, SP, CEP 03828000 
medicamentos, com renda entre 1 e 40 salários mínimos, aumentou de 2,55\% para 3,55\% entre 1988 e 1996 (Fiúza e Lisboa, 2001). Este aumento deve refletir diferentes causas: melhora de qualidade do tratamento resultante da melhora de qualidade do medicamento ${ }^{1}$ e/ou pela descoberta de novas drogas; aumento da expectativa de vida das populações; e aumento do poder de mercado por parte dos laboratórios. $^{2}$

Também seguindo uma tendência em outros países, principalmente nos desenvolvidos, em 1998 o governo brasileiro promoveu uma política de regulamentação de medicamento genérico, de modo que a partir de 1999 possibilitou sua entrada no mercado farmacêutico brasileiro. ${ }^{3}$ Esta regulamentação apresentou duas estratégias novas de políticas para a saúde no Brasil: a primeira foi a exigência institucional de teste de bioequivalência ${ }^{4}$ para a comercialização do medicamento genérico no país; a segunda foi um significativo gasto do governo com propaganda sobre a política de medicamentos genéricos. A regulamentação do medicamento genérico consiste numa atividade da Agência Nacional de Vigilância Sanitária (ANVISA), criada pela lei 9.782/99 de 1999 que também definiu o Sistema Nacional de Vigilância Sanitária. Esta autarquia pode ser vista como um órgão semelhante ao Food and Drug Administration (FDA) norte americano no que se refere às funções de controle de qualidade institucional.

Apesar da dificuldade de dissociação das causas do aumento de gastos com medicamentos, discutida acima, é razoável supor que o aumento do poder de mercado dos laboratórios é um dos seus determinantes. Considerando a estrutura concentrada dos mercados específicos de medicamento no Brasil, ${ }^{5}$ era esperado que a entrada dos medicamentos genéricos ${ }^{6}$ no país tivesse sido capaz de reduzir os custos de tratamentos individuais de alguns males ou doenças. O medicamento genérico, em geral, entra no mercado com um preço inferior ao do seu medicamento de referência. Assim, a redução no custo de tratamento pode provir de uma composição específica de consumo de genéricos e referência de tal sorte que o preço médio resultante seja menor que o preço do medicamento de referência quando era único no mercado. ${ }^{7}$

Este artigo busca verificar o efeito da entrada dos medicamentos genéricos sobre os preços dos pioneiros de marca. ${ }^{8}$ A interpretação dos resultados obtidos exige a identificação de algumas particularidades do mercado farmacêutico brasileiro, que são descritas na seqüência. A indúnstria farmacêutica brasileira gasta muito pouco em pesquisa e desenvolvimento de novos produtos (P\&D), ${ }^{9}$ a fase de desenvolvimento de novos princípios ativos ${ }^{10}$ é restrita aos países produtores. ${ }^{11} \mathrm{O}$ processo produtivo

\footnotetext{
${ }^{1}$ A literatura de preços hedônicos (Griliches, 1961) é uma das formas de tratar este fenômeno.

${ }^{2}$ Nos últimos anos houve grandes fusões e aquisições de laboratórios farmacêuticos no mundo, para maiores informações ver Danzon et alii (2004).

${ }^{3} \mathrm{O}$ medicamento genérico entrou efetivamente a partir de fevereiro de 2000 no mercado brasileiro.

${ }^{4}$ Este teste avalia se os efeitos do medicamento genérico sobre o organismo humano são idênticos aos do medicamento de referência.

${ }^{5}$ Nishijima (2003).

${ }^{6}$ Este só entra no mercado quando a patente do medicamento pioneiro tiver expirado.

${ }^{7}$ Ainda que este tenha sido aumentado após a entrada do genérico em seu mercado.

${ }^{8} \mathrm{~A}$ indisponibilidade de informações que contemplem todos os canais de distribuição de medicamentos no país não permite uma avaliação definitiva sobre as quantidades totais negociadas no mercado.

${ }^{9}$ Como o país importa os princípios ativos e o seu processo produtivo consiste apenas na sua manipulação e embalagem, poucos gastos são realizados com pesquisas das chamadas novas moléculas. Após 1990 esta característica foi reforçada porque houve o processo de abertura comercial conjugado com a manutenção do câmbio como âncora nominal durante o Plano Real, quando a taxa de câmbio se manteve apreciada, facilitando e barateando a importação não só dos princípios ativos como também dos medicamentos prontos e embalados.

${ }^{10}$ Insumo essencial para a produção de medicamentos específicos para cada tipo de doença, também denominado por fármaco ou princípio ativo. Trata-se do componente do medicamento que deve desencadear as reações bioquímicas nos indivíduos com o objetivo de curar um determinado mal.

${ }^{11} \mathrm{~A}$ remuneração deste processo deve ser garantida institucionalmente por direitos de patentes, daí decorre a pressão para os
} 
brasileiro incorpora apenas as fases finais de tratamento dos princípios ativos importados: as de manipulação dos excipientes ${ }^{12}$ e de embalagem (Bernardo e Romano, 2001). EUA, Reino Unido, Alemanha, Suíça e França são os países que concentram as inovações tecnológicas deste setor (Achilladelis e Antonakis, 2001).

O Brasil passou a respeitar as leis internacionais de patentes farmacêuticas a partir de $1996,{ }^{13}$ com a Lei de Propriedade Industrial (lei 9.279/96, ${ }^{14}$ ) que não retroagiu aos medicamentos que já estavam sendo produzidos no país antes desta data. Assim, somente os medicamentos que começaram a ser produzidos no país a partir de 1997 tiveram seu direito de patente reconhecido. Como conseqüência, a maioria dos medicamentos pioneiros de marca produzidos domesticamente se tornou potencial medicamento de referência de genéricos quando ocorreu a regulamentação.

No mercado brasileiro, antes dos genéricos, já existia concorrência em alguns mercados específicos ${ }^{15}$ propiciada pelos medicamentos "similares". Estes eram bens substitutos aos medicamentos pioneiros de marca e que tinham em sua composição o mesmo princípio ativo ${ }^{16}$ do pioneiro, além de possuírem marcas. ${ }^{17}$ Os similares surgiram com maior intensidade no país a partir de 1971 quando o governo decidiu não reconhecer patentes de medicamentos. Assim, os laboratórios que operavam domesticamente puderam produzir medicamentos patenteados em outros países, embora a legislação vigente não previsse controle de bioequivalência. Em 1976, a Vigilância Sanitária passou a controlar os medicamentos similares através da emissão de certificados de similaridade. Estes certificados podiam ser obtidos desde que os laboratórios comprovassem se tratar de um medicamento com o mesmo princípio ativo.

Em 1996, com o reconhecimento da lei de patentes farmacêuticas, ficou determinado que os laboratórios domésticos poderiam produzir similares de medicamentos pioneiros somente se as suas respectivas patentes em outros países estivessem expiradas na data de início da produção interna. ${ }^{18}$ Portanto, de modo geral, antes da lei dos genéricos a credibilidade da substituição (concorrência) de medicamentos em grande parte estava atrelada à reputação dos laboratórios.

Entre os medicamentos similares era possível identificar dois tipos: um cuja marca era um nome fantasia - geralmente conhecido do público devido às grandes campanhas publicitárias do setor - diferente da denominação de seu princípio ativo; e outro que ficou conhecido posteriormente como "pseudogenérico" ou "falso genérico", cujo nome fantasia era a própria denominação de seu princípio ativo.

Como a pré-existência dos "falsos genéricos" no mercado tendia a confundir os consumidores que buscavam os genéricos logo após a veiculação de propagandas por parte do governo - o que poderia comprometer a sua credibilidade como bens substitutos homogêneos - tal denominação de marca passou a ser proibida por lei. Deste modo, tais medicamentos foram obrigados a mudar seus nomes até setembro de 2001. Entretanto, muitos deles realizaram testes de bioequivalência e continuaram a ser comercializados no mercado como genéricos. ${ }^{19}$

países em desenvolvimento respeitem as leis internacionais de patentes. Para informações sobre produtividade e patentes ver Griliches (1989), Lanjouw e Cockburn (2000), Smith (2002), Lanjouw (1998), Grossman e Lai (2002), Kremer (1994). Para o Brasil, nem o beneficio do "efeito transbordamento" das firmas grandes para as pequenas - fenômeno ocorrido na indústria japonesa (Watanabe et alii, 2000) - se aplica, já que a fase de P\&D praticamente inexiste no país.

${ }^{12}$ Ingredientes que dão a forma e características físicas do medicamento, tais como farinha, líquidos, gel, corantes, flavorizantes, conservantes e etc. São substâncias inertes ao tratamento de um mal, destinadas a gerar a forma física do bem final, tais como: comprimidos, injeções e xaropes.

${ }^{13}$ (Bermudez et alii, 2000).

${ }^{14}$ Esta lei alterou a lei 5.772 de 1971, que excluía o respeito a patentes dos produtos químico-famacêuticos e medicamentos de qualquer espécie.

${ }^{15}$ Os mercados das classes terapêuticas.

${ }^{16}$ Podem diferir do medicamento pioneiro na forma de apresentação, na dosagem, na absorção sanguínea, tempo de ação no organismo, etc.

${ }^{17} \mathrm{O}$ medicamento genérico que não possui marca e é denominado pelo nome de seu princípio ativo.

${ }^{18}$ Estas informações foram obtidas da publicação "Assistência Farmacêutica" da Secretaria Executiva do Ministério da Saúde de Junho de 2000.

${ }^{19}$ Neste artigo os falsos genéricos que entraram no mercado como genéricos são considerados como tal, os demais são excluídos 
Este artigo avalia o efeito da entrada dos genéricos sobre os preços dos medicamentos de referência levando em conta, além das características sumariadas acima, que: a entrada dos medicamentos genéricos no mercado brasileiro ocorreu efetivamente a partir de fevereiro de 2000; a autorização para a sua produção no país foi condicionada pela realização obrigatória de teste de bioequivalência, configurando um controle de qualidade institucional inédito no país; e que o governo realizou grandes gastos publicitários sobre esta política. Além desta introdução, a seção 2 faz uma breve revisão da bibliografia sobre o tema; a seção 3 apresenta os microdados utilizados neste trabalho, assim como algumas estatísticas preliminares sobre o comportamento dos preços dos medicamentos; nas seções 4 e 5 são apresentados os modelos estimados e os seus resultados; e na última seção são apresentadas as conclusões.

\section{REVISÃO BIBLIOGRÁFICA}

Alguns estudos teóricos, considerando empresas com algum poder de mercado, avaliam o efeito da entrada de produtos genéricos em seus mercados. Em geral, apresentam o aumento de preços dos bens pioneiros em resposta à entrada dos genéricos em seus respectivos mercados, embora a possibilidade teórica de redução de preços também esteja colocada.

O trabalho de (Rosenthal, 1980) demonstra o resultado de um equilíbrio de Nash simétrico em Cournot e tem como hipótese que cada vendedor individual não é capaz de impor preços diferentes para diferentes classes de compradores: aqueles que estão sob poder de monopólio da marca e os que não estão. Neste modelo o equilíbrio de mercado é resultado da estratégia dominante da empresa pioneira de aumentar seu preço e manter apenas os consumidores leais à sua marca, quando surge concorrência em seu mercado.

(Frank e Salkever, 1991) trabalham especificamente com mercado farmacêutico e deduzem as condições de equilíbrio de um modelo de jogos de Stackelberg - com base em funções de demanda segmentada - analisando os efeitos da entrada dos genéricos sobre os preços dos medicamentos de marca. Concluem que o aumento de preços dos medicamentos de marca depois da entrada de genéricos no mercado pode ser explicado pelo comportamento de otimização do produtor do medicamento que perde a proteção da patente. Pois, de acordo com os autores, sob a hipótese de custos marginais de produção constantes, a entrada de genéricos diminui a elasticidade preço da forma reduzida da demanda.

(Kamien e Zang, 1999) fazem uma análise semelhante usando modelos estáticos calculados em dois períodos para duas situações distintas: o incumbente ${ }^{20}$ decide começar a produzir o seu próprio genérico antes de sua patente expirar, tornando-se conseqüentemente o líder do modelo de Stackelberg; e o incumbente não produz genérico nem depois da perda da patente, o que leva a um mercado típico de Cournot. Os seus resultados são condicionados pela hipótese de valores específicos atribuídos às elasticidades das demandas lineares e revelam a possibilidade de aumento de preço dos medicamentos pioneiros após a entrada dos genéricos em seus mercados.

Grabowski e Vernon (1992) realizam estudo empírico e apresentam como um dos seus resultados utilizando dados do $\mathrm{IMS}^{21}$ para 18 tipos de medicamentos orais - o fato de que a concorrência de medicamentos genéricos, que se acentuou muito no mercado americano após Waxman-Hacht Act de $1984,{ }^{22}$ tendeu a afetar positivamente a maioria dos preços dos medicamentos pioneiros por eles estudados. A explicação para o aumento do preço do medicamento de referência após a entrada do genérico em seu mercado, de acordo com estes autores, reside na segmentação da demanda por medicamento em dois tipos de consumidores, um mais sensível a preços - em geral hospitais - e outro fiel à marca.

da amostra.

${ }^{20}$ Este termo é usado por (Aghion e Howitt, 1992) para discriminar um empresário capitalista que realiza uma inovação tecnológica no mercado.

${ }^{21}$ International Medical Statistics.

${ }^{22}$ Nos EUA os genéricos foram introduzidos em 1973, mas foi somente depois de 1984 que ocorreram entradas de tais medicamentos em volume significativo. 
Para o mercado brasileiro, Fiúza e Lisboa (2001) mostram empiricamente que a concorrência prévia - propiciada pelos medicamentos similares e derivada da disputa entre laboratórios privados - também causava efeitos positivos sobre os preços dos medicamentos referência. Para este fim, estimaram modelos de efeitos fixos e aleatórios utilizando dados de medicamentos de marca, líderes de mercado, e de medicamentos similares entre os períodos de 1995 e 1999.

\section{DADOS E ESTATÍSTICAS PRELIMINARES}

Todos os dados usados neste estudo foram obtidos secundariamente através do Ministério da Saúde, as fontes primárias são descritas na seqüência. A identificação dos medicamentos genéricos - as datas das autorizações para a produção e comercialização em resposta aos laboratórios com produtos aprovados pelo teste de bioequivalência, tipos de apresentação, o medicamento pioneiro ao qual se refere e as características específicas - foi obtida pela coleta diária de autorizações para a produção publicadas no Diário Oficial da União (DOU) e ANVISA. Até fevereiro de 2002, 1892 apresentações de medicamentos genéricos de diferentes laboratórios obtiveram autorização da ANVISA para a entrada no mercado.

As seguintes informações foram obtidas das publicações anuais do International Medical Statistics (IMS): preços médios; ${ }^{23}$ data de lançamento no mercado; quantidade comercializada; classe terapêutica; e a classificação por consumo, se uso livre ou controlado dos medicamentos de referência e demais medicamentos. Este banco possui 4 anos $^{24}$ de informações anuais entre 1998 e 2001, sendo que em 1998 contava com 7585 apresentações de medicamentos e em 2001 com $8937 .^{25}$

Informações sobre preços em moeda nacional foram obtidas da Associação Brasileira de Comércio Farmacêutico (ABCFARMA). Este banco contava com 17.675 diferentes apresentações de medicamentos ${ }^{26}$ no início do ano de 2002. Este banco possui informações mensais, a partir de janeiro de 1998, sobre: laboratórios produtores; marca; se possui tarja vermelha ou preta - ou seja, se só pode ser consumido com receita médica ou se além da receita obrigatória tem seu uso controlado; e valores nominais correntes do preço fábrica e do preço consumidor. ${ }^{27}$ Informações sobre índices de preços gerais e do setor farmacêutico, usadas para deflacionar dos preços dos medicamentos, são do INPC do IBGE.

A unidade usada nas estimativas corresponde a da apresentação do medicamento. Nota-se que cada medicamento pode ter mais de uma forma de apresentação. O cruzamento entre os bancos de dados foi possível porque em cada um se identificou todas as apresentações dos medicamentos que os compunham. A apresentação de um mesmo medicamento pode diferir: na embalagem, por exemplo, no número diferente de comprimidos; no corpo do excipiente, por exemplo, comprimido ou solução aquosa; na dosagem, por exemplo, $100 \mathrm{mg}$ ou $500 \mathrm{mg}$ de princípio ativo, etc. Assim, o medicamento Depakote, por exemplo, possui 3 apresentações - frasco de 20 comprimidos de $250 \mathrm{mg}$, frasco com 20 comprimidos de $500 \mathrm{mg}$ e caixa sprinkle com 30 comprimidos de $250 \mathrm{mg}$ - que são considerados, neste trabalho, como 3 medicamentos distintos para efeito de comparação no tempo. A localização e cruzamento destas informações despenderam muito tempo de trabalho ${ }^{28}$ e foram necessárias porque cada banco de dados utiliza códigos próprios para as apresentações de medicamentos, que diferem entre

${ }^{23}$ Derivados da divisão do faturamento em dólares pela quantidade comercializada Este procedimento é muito comum na literatura internacional que utiliza os dados do IMS.

${ }^{24}$ Os cadernos disponíveis continham informações mensais dos últimos 12 meses das seguintes datas de publicação: setembro de 1998; dezembro de 1999; junho de 2000; outubro de 2000; e maio de 2001. Com base nestas informações foram montados dados anuais de junho do ano $t$ até maio do ano $t+1$.

${ }^{25}$ Apesar do crescimento no número de apresentações, houve várias descontinuidades de apresentações.

${ }^{26}$ Este não reflete adequadamente o número de medicamentos disponíveis no mercado, pois os que deixaram de ser comercializados continuam com o código constando neste banco.

${ }^{27} \mathrm{~A}$ diferença entre eles corresponde à margem de lucro das farmácias. O preço fábrica é o valor que as farmácias pagam aos laboratórios e o preço ao consumidor é o preço máximo que a farmácia deve cobrar.

${ }^{28}$ Agradeço à inestimável colaboração de Cecília Haffers, Reus Coutinho e Nilva Rocha. 
si, além de existirem problemas como códigos duplicados para medicamentos diferentes num mesmo banco, não sendo possível o cruzamento imediato dos bancos.

O resultado do cruzamento dos bancos descritos acima culminou num painel com dados anuais de 1999 até 2001 com 4557 apresentações de medicamentos. Os dados mensais foram anualizados da seguinte maneira: ano 1999, de junho de 1998 até maio de 1999; ano 2000, de junho de 1999 até maio de 2000; e ano 2001, de junho de 2000 até maio de 2001. Foram incluídas apenas apresentações de medicamentos disponíveis ao longo de todo o período de tempo investigado, apresentações descontinuadas no mercado foram excluídas da amostra. Este painel, entretanto, não contém todas as informações sobre todos os seus medicamentos contemplados, por exemplo, grande parte dos medicamentos não possui informação sobre sua data de lançamento no mercado, tendo sido usado missing para as informações indisponíveis. ${ }^{29}$ Por esse motivo, cada tabela de resultados apresentada neste trabalho contém o número de observações (número de medicamentos vezes o número de anos) utilizadas nas estimativas específicas descritas, conforme a disponibilidade de informações para cada modelo.

Importante notar que as informações disponíveis sobre medicamentos se referem exclusivamente aos comercializados em farmácias, principal canal de distribuição de medicamentos no país, não incorporando a compra por hospitais e demais unidades de atendimento médico. Os bancos de dados primários também apresentam esta característica. Assim, são eliminados os potenciais consumidores com algum poder de mercado, restando os consumidores pulverizados.

A avaliação preliminar do painel sugere algumas diferenças de comportamento entre a média dos preços dos medicamentos que se tornaram referência de genéricos em relação à média dos preços dos demais medicamentos. As diferenças de variações entre essas médias de preços são mostradas na tabela 1, que apresenta tanto a comparação de preços medidos em reais constantes de janeiro de 1999 como também em dólares correntes.

Tabela 1 - Diferenças em diferenças do efeito da entrada de genéricos sobre a média dos preços dos medicamentos de marca

\begin{tabular}{rrrrrrr}
\hline \multicolumn{7}{c}{ Preços em dólares correntes } \\
Medicamentos de Marca & 1999 & 2000 & 2001 & {$[2001]-[2000]$} & {$[2001]-[1999]$} & n. obs. \\
Referência (R) & 12,93 & 13,44 & 12,33 & $-1,11(-5,37)$ & $-0,60(-4,46)$ & 504 \\
Não-Referência (NR) & 8,43 & 8,78 & 8,22 & $-0,56(-20,66)$ & $-0,21(-13,62)$ & 4467 \\
(R)-(NR) & 4,5 & 4,66 & 4,11 & $-0,55(-3,94)$ & $-0,39(-1,80)$ & \\
\multicolumn{7}{c}{ Preços em reais de 1999} \\
Medicamentos de Marca & 1999 & 2000 & 2001 & {$[2001]-[2000]$} & {$[2001]-[1999]$} & n. obs. \\
Referência (R) & 22,98 & 22,95 & 22,29 & $-0,66(-3,26)$ & $-0,69(-1,70)$ & 453 \\
Não-Referência (NR) & 14,63 & 14,61 & 14.18 & $-0,43(-16,44)$ & $-0,43(-5,52)$ & 5361 \\
(R)-(NR) & 8,35 & 8,34 & 8,11 & $-0,23(-2,80)$ & $-0,26(-1,40)$ & \\
\hline
\end{tabular}

Estatística t de teste de igualdade de média entre parênteses

A comparação por testes de médias revela que: a) as médias dos preços dos medicamentos que se tornaram referência de genéricos são bastante superiores às dos demais medicamentos; b) os medicamentos em geral apresentaram uma pequena queda na média de seus preços em 2001. Os preços em dólares inicialmente aumentaram entre 1999 e 2000, reduzindo em 2001, enquanto os preços em reais sempre caíram entre estas datas. A diferença de comportamento reflete o impacto da variação nominal do câmbio ocorrida no período, decorrente da mudança do regime cambial brasileiro e o não deflacionamento dos preços em dólares; c) os preços dos medicamentos que não se tornaram referência

\footnotetext{
${ }^{29}$ Esta possibilidade surge como decorrência do pacote STATA que em suas estimativas utiliza todas as informações disponíveis e descarta as indisponíveis.
} 
apresentaram uma pequena queda em 2001, já a queda dos preços dos medicamentos que passaram a enfrentar os genéricos foi maior, como revelam as diferenças das diferenças de médias de preços ao longo do tempo entre estes dois tipos de medicamentos. Estas médias, entretanto, não devem ser tomadas como conclusivas, pois são medidas muito sensíveis a valores extremos. Para uma conclusão mais consistente do fenômeno, uma análise mais criteriosa do comportamento dos preços dos medicamentos após a entrada dos genéricos no mercado é necessária, o que é apresentado na seção 4.

A análise da relação entre a idade dos medicamentos - variável criada, com referência no ano de 2001, a partir da informação da data de entrada do medicamento no mercado - e o seu respectivo preço em reais de 1999 revela que os medicamentos lançados há mais tempo no mercado tendem a apresentar preços menores, em média, do que aqueles lançados mais recentemente, figura 1. Embora mostre preços de diferentes medicamentos, ${ }^{30}$ gerando uma medida imperfeita do padrão do ciclo de vida dos medicamentos, este gráfico sugere uma idéia do que seria o padrão da relação entre preços e idade de medicamentos no Brasil. Observa-se que o preço do produto seria alto no lançamento, crescendo rapidamente um pouco mais até atingir seu pico, decrescendo suavemente, a partir daí, com a maturidade do produto no mercado. Este comportamento sugere que o período de penetração dos novos medicamentos no mercado brasileiro é bastante reduzido, diferente dos Estados Unidos onde os novos lançados experimentam um significativo período de declínio de preços no início do seu ciclo de vida (Griliches e Cockburn, 1993) e (Berndt et al., 1992). ${ }^{31}$ No gráfico, os medicamentos mais novos, com zero ou um ano de idade, e com menores preços, são os medicamentos genéricos, pois no corte da amostra foram excluídos os medicamentos de marca que entraram no mercado após junho de 1998.

A figura 2 corrobora a idéia descrita acima, pois mostra a relação entre os preços dos medicamentos e suas respectivas idades elevadas ao quadrado. Ao invés de se verificar uma parábola com a concavidade voltada para baixo, como se esperaria no caso em que a chegada da maturidade fosse mais demorada, exigindo que preços menores fossem cobrados por um período mais longo após o lançamento do produto no mercado, o gráfico sugere uma hipérbole.

Uma possível explicação para um medicamento já ser lançado no mercado brasileiro com o preço alto $^{32}$ seria o fato das novas drogas chegarem com atrasos nos países que não são produtores de medicamentos e cujos mercados são secundários. Nos países produtores, que são os que também apresentam os maiores mercados consumidores, a competição é mais acirrada e a rápida inovação tecnológica é uma necessidade de sobrevivência. Mas, em países periféricos, as subsidiárias ou transformam o princípio ativo importado numa forma física comercializável ou simplesmente importam o medicamento, de modo que as inovações tendem a ocorrer com atraso. Assim, quando os novos medicamentos entram nesses mercados, os seus benefícios já são amplamente conhecidos pela experiência prévia do seu uso nos países produtores e por causa dos enormes gastos com publicidade, que caracterizam esta indústria conforme Hurwitz e Caves (1988), prescindindo da fase inicial de preços baixos para a penetração no mercado.

\section{PREÇOS DOS MEDICAMENTOS DE REFERÊNCIA: MODELO DE DIFERENÇA EM DIFE- RENÇAS}

Para verificar empiricamente se a entrada dos medicamentos genéricos afetou - e de que maneira - os preços dos medicamentos pioneiros de marca que se tornaram referência foi adotada a mesma

${ }^{30} \mathrm{O}$ ideal seria acompanhar o mesmo medicamento ao longo de um longo período de tempo.

${ }^{31}$ Veja também McClellan (1995). No caso dos medicamentos, episódios como da Talidomida ilustram a dificuldade inicial da penetração de uma nova molécula (princípio ativo) no mercado farmacêutico.

${ }^{32}$ Para estudo deste fenômeno seriam necessárias séries de preços de medicamentos que permitissem a realização de coortes, entretanto, dada esta impossibilidade usamos os resultados obtidos apenas como auxilio na análise dos modelos estimados na próxima seção. 
Figura 1 - Dispersão entre preços e idade dos medicamentos no mercado brasileiro

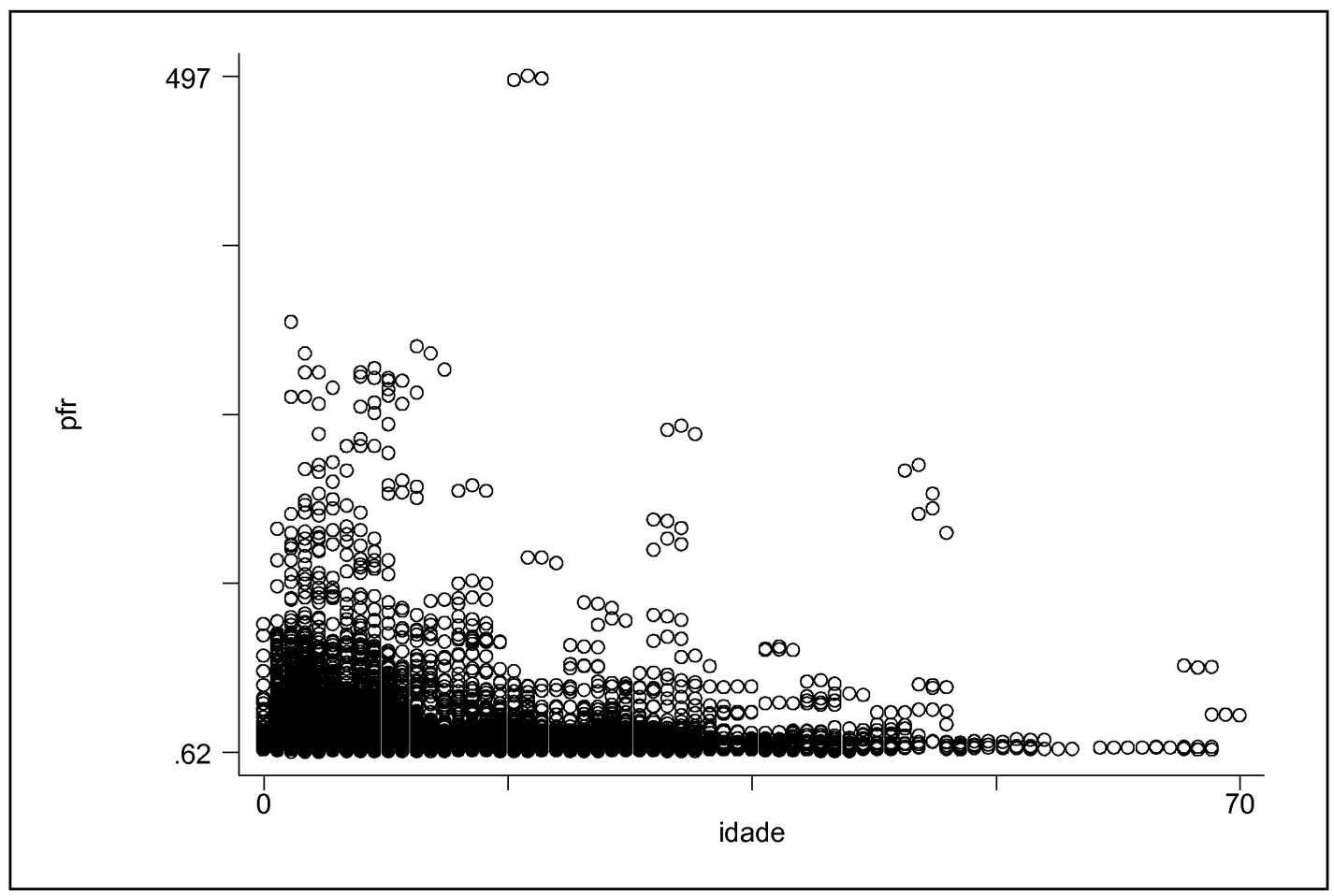

estratégia econométrica utilizada por Pavcnik (2000). ${ }^{33}$ Esta consistiu em estimar modelos lineares de diferença em diferenças (DD), que avaliam as diferenças entre as médias de dois grupos e as suas respectivas diferenças ao longo do tempo. Para os medicamentos, foram comparadas as diferenças de variações dos preços dos pioneiros que se tornaram referência de genéricos com as variações dos preços dos medicamentos que não enfrentaram esta concorrência ${ }^{34}$ ao longo do tempo, como forma de avaliar se houve diferenças nas decisões dos produtores sobre os preços destes dois grupos. Em particular, buscou-se verificar se a escolha de preços dos produtores de medicamentos pioneiros de marca, que passaram a sofrer concorrência dos genéricos sob a égide das garantias institucionais, ${ }^{35}$ foi semelhante à adotada em relação aos medicamentos "similares" apontada por Fiúza e Lisboa (2001). ${ }^{36}$

O uso de modelos DD permite que sejam feitas análises temporais dos efeitos de intervenções (Lee, 2005). Considerando a intervenção como sendo a entrada dos medicamentos genéricos em alguns mercados, pode-se considerar o grupo dos tratados como sendo o dos medicamentos pioneiros de marca que sofreu esta concorrência. $\mathrm{O}$ uso do grupo dos não tratados permite controlar os efeitos comuns a todos os medicamentos não relacionados com a entrada dos genéricos, tais como as políticas de controles de preços iniciada no final de 2000 e implantadas a partir de janeiro de 2001. Note, entretanto, que a amostra utilizada neste estudo cobre o período de junho de 1998 até maio de 2001, mas as

\footnotetext{
${ }^{33} \mathrm{~A}$ autora usou a técnica para avaliar outro fenômeno econômico. No presente trabalho a técnica foi adaptada para o caso dos medicamentos.

${ }^{34} \mathrm{Na}$ verdade, incluem todos os demais medicamentos, inclusive os similares.

${ }^{35}$ Dada pelo teste de bioequivalência e pela criação da ANVISA.

${ }^{36}$ Ainda que a metodologia econométrica utilizada tenha sido diferente.
} 
Figura 2 - Dispersão entre preço e idade ao quadrado dos medicamentos

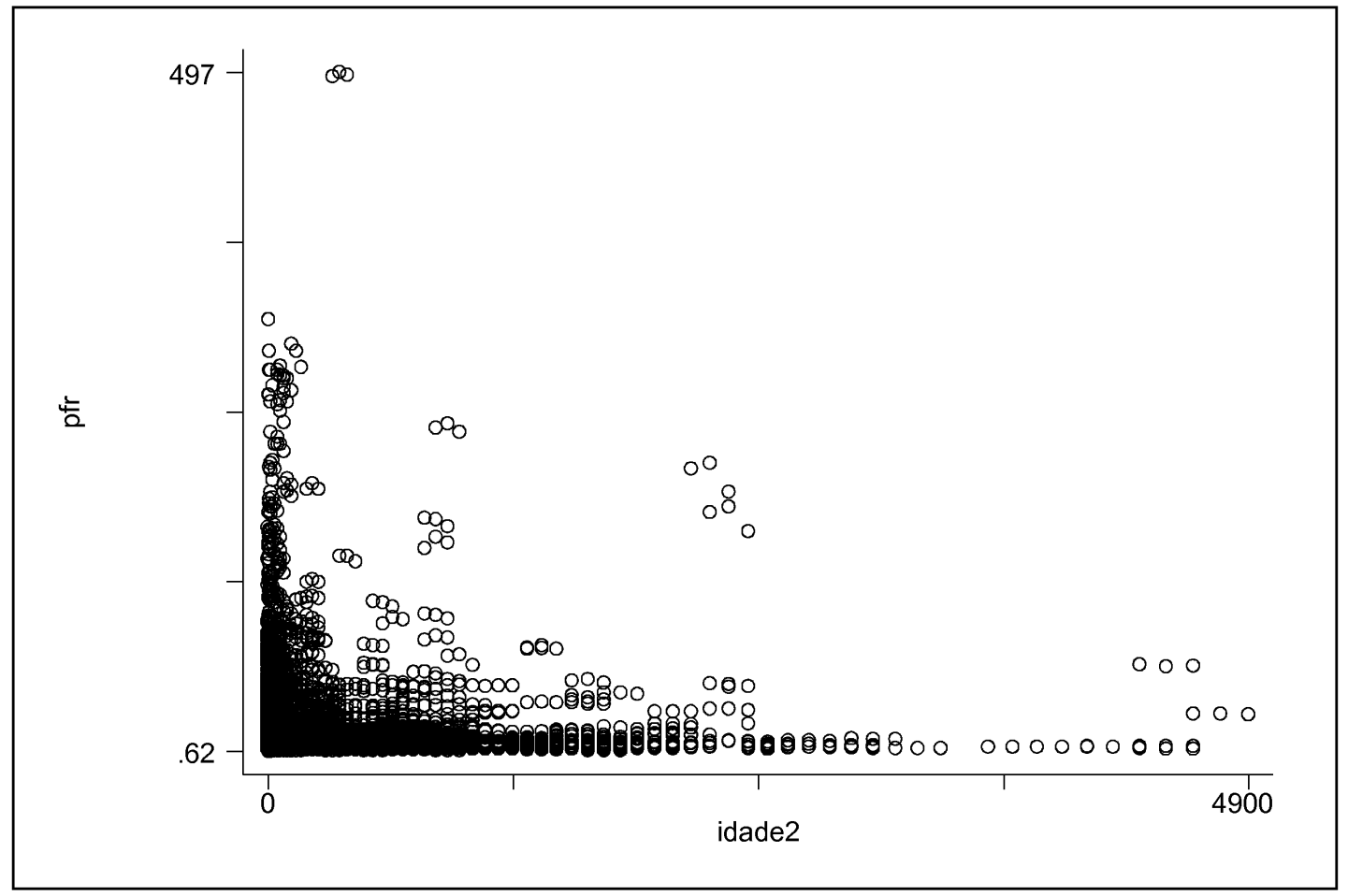

competências da CAMED (Câmera de Medicamentos) como órgão regulador e de fiscalização de preços só foram instituídas em março de 2001. Deste modo, na amostra usada somente os últimos 5 meses que compõem o ano de 2001 estão sob o efeito do congelamento de preços, que mesmo assim foi aplicado a todos os medicamentos. A inclusão de variáveis dummies de tempo permite captar os efeitos de mudanças macroeconômicas, tais como da mudança de regime cambial brasileiro em 1999. Além disso, como se trabalha com as diferenças no tempo, os efeitos fixos dos medicamentos individuais são controlados, evitando potenciais problemas de endogeneidade.

Os modelos DD são também modelos de intervenção before-after, pois permitem que sejam feitas análises da variável de interesse (preço do medicamento pioneiro, neste caso) antes e depois da intervenção ocorrer (Lee, 2004). Tendo em conta que o painel corresponde aos anos de 1999 até 2001 e a entrada dos genéricos se iniciou em fevereiro de 2000, o uso deste modelo de intervenção é adequado ao período da amostra dos medicamentos pesquisados.

A teoria econômica, que sugere a relação de variáveis a serem investigadas empiricamente neste estudo, provém da oferta em mercados com poder preço (não competição perfeita). Como nestes mercados os produtores são price makers, é usual considerar uma estrutura de investigação dos determinantes dos preços pelo lado da oferta. Fiúza e Lisboa (2001), Hurwitz e Caves (1988) e Grabowski e Vernon (1992) adotam este referencial teórico. Deste modo, o modelo linear de diferenças em diferenças pode ser descrito por:

$$
p_{i t}=\alpha_{0} \phi_{i}+\alpha_{1} \mu_{i t}+\alpha_{2} \eta_{i t}+\alpha_{3}(\mu * \eta)_{i t}+\alpha_{4} Z_{i t}+v_{i t}
$$

Sendo $p_{i t}$ o vetor de preços de cada medicamento $i$ no período $t$; $\phi_{i}$ é um vetor de constantes que 
capta as diferenças específicas de cada medicamento $i$ que não varia no tempo, os efeitos fixos; $\mu_{i t}$ é um vetor de dummies de tempo; $\eta_{i t}$ é um vetor de dummies que descreve se um medicamento é ou não um referência ${ }^{37} ; \mu * \eta$ é um vetor de variáveis que são interações entre as dummies de tempo e as dummy de entrada de medicamento genérico; $Z_{i t}$ é um vetor de características dos medicamentos que variam com o tempo, tais como idade, idade ao quadrado, a quantidade vendida de cada medicamento; e $v_{i t}$ é um vetor de distúrbios aleatórios com média zero e que atende as condições de ortogonalidade.

O modelo linear DD da equação (1) foi estimado, tendo como variável dependente os preços em reais constantes de 1999, pelos seguintes métodos: a) mínimos quadrados ordinários (MQO) com dummies de classes terapêuticas em seu nível mais agregado - nível 1 da Anatomical Therapeutic Classification (ATC) da EphMRA, ${ }^{38}$ as estimativas foram omitidas nas tabelas mas a maioria se mostrou significativa - para levar em conta as diferenças entre medicamentos de classes distintas; b) modelo de efeito fixo (MEF), quando as classes terapêuticas em seu nível mais desagregado (nível 4 da ATC) foram consideradas efeitos fixos; c) Estimativas robustas - ${ }^{39}$ corrigidas pelo problema de heteroscedaticidade - de MEF com o fixo considerado por medicamento ${ }^{40}$; d) Mesmo modelo do item anterior excluindo a variável explicativa quantidade de medicamento; e) MEF usando variável instrumental.

Tabela 2 - Modelos de Diferenças em diferenças: preços em reais

\begin{tabular}{llllll}
\hline $\begin{array}{l}\text { Modelos } \\
\text { Estimados }\end{array}$ & $\begin{array}{l}\text { a) MQO com } \\
\text { dummies de } \\
\text { classe terapêu- } \\
\text { tica nível 1 }\end{array}$ & $\begin{array}{l}\text { b) MEF i(classe } \\
\text { nível 3) }\end{array}$ & $\begin{array}{l}\text { c) } \\
\text { i(medicam) } \\
\text { robusto }\end{array}$ & $\begin{array}{l}\text { MEF } \\
\text { exclusive quanti- } \\
\text { dade }\end{array}$ & $\begin{array}{l}\text { e) } \\
\text { i(medicam) }\end{array}$ \\
\hline \multicolumn{7}{l}{ Variável dependente: preço em reais constantes de 1999 } & & MEF-IV \\
\hline quant. & $-0.0034(-11.16)^{*}$ & $-0.0023(-9.57)^{*}$ & $-0.0004(-4.99)^{*}$ & - & $-0.002(-2.98)^{*}$ \\
idade & $-0.7195(-13.73)^{*}$ & $-0.4375(-10.15)^{*}$ & $-0.0558(-2.72)^{*}$ & $-0.0582(-2.85)^{*}$ & $-0.044(-2.14)^{*}$ \\
idade2 & $0.0110(8.04)^{*}$ & $0.00702(6.85)^{*}$ & $0.0047(4.69)^{*}$ & $0.0048(4.80)^{*}$ & $0.0041(4.06)^{*}$ \\
refx & $8.8834(7.38)^{*}$ & $8.8400(11.97)^{*}$ & Dropped & Dropped & Dropped \\
trf2 & $-0.25145(-0.43)$ & $-0.1443(-0.40)$ & $0.0098(0.26)$ & $0.0184(0.50)$ & $-0.0194(-0.49)$ \\
trf4 & $-0.29867(-0.05)$ & $-0.0144(-0.04)$ & $-0.0190(-0.59)$ & $-0.0109(-0.35)$ & $-0.042(-1.25)$ \\
trf6 & $-0.5626(-0.10)$ & $-0.1270(-0.40)$ & $-0.2809(-8.40)^{*}$ & $-0.2706(-8.32)^{*}$ & $-0.3109(-8.41)^{*}$ \\
cte & $49.905(17.40)^{*}$ & $19.196(11.83)^{*}$ & $15.715(54.68)^{*}$ & $15.580(55.77)^{*}$ & $16.19(46.11)^{*}$ \\
obs. & 13501 & 13501 & 13501 & 13501 & 13501 \\
\hline
\end{tabular}

* estatisticamente significante a 5\% de confiança e entre parênteses as estatísticas de teste.

Os modelos estimados sugerem que os preços dos medicamentos decrescem com seus respectivos tempo de lançamento no mercado - ou seja, com sua idade - e crescem com o quadrado desta variável, em acordo com a relação entre preço e idade do medicamento discutida na seção 3. O sinal positivo do coeficiente associado à variável refx nos modelos a e $b^{41}$, que descreve se o medicamento se tornou referência de genérico até 2001, reflete o fato de que estes medicamentos possuem preços mais altos que os demais, em acordo com as evidências da seção 3.

O sinal positivo do coeficiente associado à variável refx nos modelos a e b , que descreve se o medicamento se tornou referência de genérico até 2001, reflete o fato de que estes medicamentos possuem preços mais altos que os demais, em acordo com as evidências da seção 3.

\footnotetext{
${ }^{37}$ Seu valor é 1 se o medicamento se torna referência entre 1999 e 2001 e 0, caso contrário.

${ }^{38}$ European Pharmaceutical Marketing Reserting Association.

${ }^{39}$ Utilizou-se o estimador da variância de Huber/White que gera erros padrões consistentes ainda que os resíduos não sejam identicamente distribuídos.

${ }^{40} \mathrm{O}$ teste de Hausman indicou que o MEF era mais adequado que modelo de efeito aleatório.

${ }^{41}$ Para os demais modelos é eliminada por ser fixa no tempo.
} 
Nos modelos da tabela 2 , as variáveis trfs - que consistem na interação entre as dummies de entradas de genéricos no mercado ao longo do tempo e as dummies de tempo - indicam que no ano de $2001^{42}$ houve uma queda significativa dos preços dos medicamentos que passaram a enfrentar concorrência de genéricos. Pois, as variáveis com número par estão associadas aos medicamentos que se tornaram referência. No modelo DD estimado, equação 1, estas variáveis descrevem as diferenças de variações de preços entre os dois grupos de medicamentos, como o seu sinal é negativo indica que o grupo que se tornou referência de genérico teve uma queda mais acentuada de preços que os demais, uma vez que a tendência foi de redução de preços. Deve-se observar, entretanto, que estas variáveis cruzadas são bastante correlacionadas com as próprias dummies de tempo e de entrada de genéricos, o que faz com que sejam eliminadas ${ }^{43}$ para evitar problema de singularidade de matriz. Deste modo, a significância da variável trf6 capta também a tendência de queda de preços no tempo controlada pela entrada de genéricos nos mercados.

Considerando que as estimativas são dos "determinantes da oferta" tem-se o problema potencial de endogeneidade nos modelos estimados, pois preço e quantidade são determinados simultaneamente no mercado. A simples exclusão da variável quantidade no modelo não altera significativamente os valores dos coeficientes estimados, modelo d na tabela 2 , conforme teste de especificação de Hausman. A omissão da quantidade negociada no mercado pôde ser considerada porque o viés que causa possui valor contrário ao do coeficiente estimado de interesse, que corresponde ao coeficiente associado à variável que capta a entrada dos genéricos no mercado ao longo do tempo. Levando em conta que a quantidade é negativamente correlacionada com preço e negativamente correlacionada com a entrada de genéricos tem-se um viés de omissão positivo. Assim, como a variável que capta a entrada dos genéricos no tempo apresentou sinal negativo, apesar do potencial viés positivo, seu resultado pode ser indicativo.

Apesar disso, foi estimado um modelo com variável instrumental - modelo $e$ da tabela 4 - que utiliza a importância relativa de cada medicamento no faturamento total dos seus respectivos laboratórios como instrumento. A idéia deste instrumento é a de que cada laboratório produz uma grande quantidade de medicamentos em diferentes classes terapêuticas, de modo que o faturamento em cada medicamento específico tende a ser aproximadamente homogêneo nos quatro ou cinco medicamentos mais vendidos. Deste modo, o faturamento relativo do medicamento não possui uma relação estatística imediatamente direta com a quantidade ofertada. Pois, ao considerar a medida da importância relativa do faturamento do medicamento para o laboratório cria-se uma medida de ordenação pouco relacionada com oferta, mas que guarda relação com a demanda. Embora esteja longe de ser um instrumento adequado, porque não é uma informação provinda genuinamente dos demandantes, o seu uso pode ser justificado pela indisponibilidade de dados sobre demanda de medicamentos no país, especialmente no nível de desagregação aqui utilizado.

A seqüência de modelos apresentada busca mostrar a importância das diferenças específicas entre os medicamentos, que são significativas, de tal maneira que não basta introduzir controles por classe terapêutica - ainda que seja em seu nível mais desagregado. Torna-se, necessário considerar o efeito fixo por medicamento para que as estimativas sejam adequadas. Entretanto, numa análise crítica mais profunda $^{44}$ é necessário levar em consideração que ao comparar preços de diferentes apresentações de medicamentos está sendo feita uma comparando de preços de bens diferentes. Por exemplo, com relação a uma caixa com mais comprimidos que outra é natural que a magnitude do aumento de preços da primeira seja maior que a da caixa com menos comprimidos. Para levar em conta essas diferenças,

\footnotetext{
${ }^{42}$ Lembrando que nestes dados o ano de 2001 vai apenas até o mês de maio, torna-se interessante tal análise, por refletir o período em que o setor operou com a maior liberdade de preços. A partir desta data o governo fez um acordo de desoneração de certos impostos em troca de certo controle de preços, o que forçou uma queda geral nos preços do setor, sem que necessariamente as relações entre preços relativos tenham sido mantidas.

${ }^{43}$ O próprio programa utilizado, STATA, para a estimação faz a eliminação.

${ }^{44}$ Agradeço a um parecerista anônimo pelas sugestões.
} 
tendo a indisponibilidade de dados adicionais como limite, é preciso estimar o modelo DD utilizando o logaritmo natural das variáveis, tabela 3.

Tabela 3 - Modelos de Diferenças em Diferenças - logaritmo das variáveis

\begin{tabular}{llll}
\hline $\begin{array}{l}\text { Modelos } \\
\text { Estimados }\end{array}$ & $\begin{array}{l}\text { c) MEF i(medicam) } \\
\text { Robusto }\end{array}$ & $\begin{array}{l}\text { d)MEF i(medicam) } \\
\text { Exclu. Quantidade }\end{array}$ & e) MEF-IV i(medicam) \\
\hline \multicolumn{4}{l}{ Variável dependente: preço em reais constantes de 1999 } \\
\hline Quant. & $-0.0141(-4.25)^{*}$ & - & $-0.0070(-3.65)^{*}$ \\
Idade & $-0.0264(-6.21)^{*}$ & $-0.0317(-6.55)^{*}$ & $-0.039(-6.46)^{*}$ \\
trf2 & $0.00101(0.84)$ & $0.0009(0.44)$ & $-0.002(-0.17)$ \\
trf4 & $-0.0037(-3.79)^{*}$ & $0.0042(4.33)^{*}$ & $0.0031(3.02)^{*}$ \\
trf6 & $-0.0080(-8.49)^{*}$ & $-0.0065(-7.06)^{*}$ & $-0.008(-7.08)^{*}$ \\
Cte & $2.3600(122.75)^{*}$ & $2.312(200.90)^{*}$ & $2.374(176.65)^{*}$ \\
obs. & 13432 & 13433 & 12776 \\
\hline
\end{tabular}

* estatisticamente significante a 5\% de confiança e entre parênteses as estatísticas de teste.

As estimativas robustas dos modelos $c, d$ e $e$ em seus logaritmos naturais, tabela 3 , revelam que os principais resultados obtidos com as variáveis em nível, tabela 2 , são mantidos, a menos da variável de interação trf4 que passa a ser estatisticamente significativa com coeficiente positivo. Sugerindo que a entrada dos genéricos nos mercados específicos fez com que, no momento de sua entrada - ano de 2000 - os preços dos medicamentos de referência tenham aumentado e posteriormente - em 2001 tenham diminuído. Deve-se notar que não foi incluído o logaritmo da variável idade ao quadrado nos modelos estimados, pois ao fazer a transformação esta se torna combinação linear do logaritmo da variável idade, causando singularidade na matriz das equações do modelo.

Os resultados das estimativas acima sugerem uma possível melhora de bem estar social e se diferenciam tanto dos resultados obtidos para o mercado americano como dos resultados obtidos pela concorrência dos medicamentos pioneiros com os seus similares no mercado brasileiro. Vale notar que mesmo com a escassez de dados disponíveis para a análise, contando com uma amostra de apenas um ano e meio ${ }^{45}$ de existência de medicamentos genéricos no mercado, foi possível obter algumas informações preliminares sobre comportamento dos preços dos medicamentos referência e genéricos no mercado brasileiro. Trabalhos futuros, entretanto, serão importantes para avaliar se a tendência de queda dos preços dos medicamentos de referência foi mantida. No entanto, uma dificuldade adicional é que a partir de maio de 2001 o setor farmacêutico passou a ser regulado de maneira mais efetiva sob a égide da CAMED, que passou a ter poderes de fiscalização e regulação em março de 2001.

Na seção 5 é apresentado outro exercício econométrico - usando uma amostra com período mais longo, mas com um número menor de medicamentos - que busca avaliar o impacto do número de medicamentos genéricos sobre os preços dos seus respectivos medicamentos referência. 0 exercício se diferencia do realizado nesta seção porque a amostra contém somente medicamentos que se tornaram referência de genéricos e pelo objetivo que consiste em verificar, dada a entrada do medicamento genérico no mercado, qual é o impacto de diferentes quantidades de genéricos disponíveis.

\footnotetext{
${ }^{45}$ Têm-se um ano de amostra de medicamentos em que ainda não havia genéricos, 1999, e um ano e meio de amostra de medicamentos de marca convivendo com genéricos. O banco de dados da ABCFARMA é mais recente, contendo preços até dezembro de 2002. Mas com o cruzamento de informações necessário para obter as variáveis usadas nesta seção foi necessário limitar a amostra pelo menor número de informações do IMS disponíveis.
} 


\section{COMPORTAMENTO DOS PREÇOS DOS MEDICAMENTOS DE MARCA CONFORME O NÚ- MERO DE MEDICAMENTOS GENÉRICOS QUE ENTRAM NO MERCADO}

Inspirado nos modelos econométricos de longo prazo estimados por Caves et alii (1991), esta seção apresenta um estudo sobre o comportamento dos preços dos medicamentos de marca que passaram a enfrentar concorrência crescente dos medicamentos genéricos no Brasil. Isto é, uma vez que existe medicamento genérico no mercado de um bem, qual o efeito do aumento do número de produtores de genérico sobre o preço do pioneiro. A análise para o Brasil, entretanto, é de curto prazo e leva em conta que a partir da entrada em vigor da lei de genéricos a maioria dos medicamentos pioneiros ${ }^{46}$ pôde se tornar referência de genéricos simultaneamente.

Caves et alii (1991) avaliam o comportamento dos preços dos medicamentos pioneiros quando cresce o número de medicamentos genéricos concorrendo em seus respectivos mercados, com base numa amostra de 11 anos de informações sobre o mercado farmacêutico americano. Estimam modelos em diferenças dos logaritmos das variáveis usando modelos de variável instrumental que, de acordo com os autores, podem ser avaliados como uma forma simples de elasticidade preço constante. A variável explicada é a variação de preços dos medicamentos de marca a cada período, $p_{i t}$. As variáveis explicativas são: margem de lucro como função da elasticidade da demanda do consumidor frente ao medicamento de marca $i$ no período $t$, medida por variáveis referentes ao tempo de exposição no mercado, tais como idade, e por variáveis relacionadas ao grau de concorrência no mercado, tal como o número de genéricos de cada medicamento de marca em cada período $t$; custo marginal de produção, $\mu_{i t}$, que por falta de informação sobre custos é decomposto em características específicas dos medicamentos, efeito fixo, efeitos agregados da indústria e dummies de tempo; e erro aleatório não observado, $\varepsilon_{i t}$. Os autores concluem que os preços dos medicamentos de marca apresentam um pequeno declínio com o aumento do número de genéricos do mesmo medicamento de marca.

O intuito deste estudo foi verificar, para o caso brasileiro, a possibilidade de captar algum efeito do aumento do número de produtores de medicamentos genéricos de um mesmo medicamento de referência sobre o seu preço. Tal estudo, entretanto, foi realizado sem perder de vista que: os dados não eram ideais e que o período de tempo de existência dos genéricos no mercado brasileiro é ainda muito reduzido - apenas 4 anos de amostra, dos quais apenas 3 anos apresentam efetivamente medicamentos genéricos - o que não permite uma análise de longo prazo; o final do período da amostra compreende regulação de preços no mercado, o que, ao contrário da seção anterior, não é controlado pelo grupo de medicamentos que não sofreu a concorrência dos genéricos.

Para este procedimento foi realizado um cruzamento de informações dos bancos de dados disponíveis que resultou em 162 apresentações de medicamentos de referência, para um período de quatro anos, de 1999 até 2002, usando dados mensais com intervalos de seis meses. ${ }^{47}$ Como nesta amostra só foram incluídos medicamentos de referência, foi pressuposto que as dummies de tempo são capazes de captar os efeitos macroeconômicos comuns aos preços dos demais medicamentos.

O modelo linear estimado pode ser descrito por:

$$
p f_{i t}=\alpha_{i}+\beta_{1} \text { lagentry }_{i t}+\beta_{2} \text { ngene }_{i t}+\beta_{3} \text { idade }_{i t}+\beta_{4} i d a d e 2_{i t}+\beta_{4} \mu_{t}+\varepsilon_{i t}
$$

$O$ vetor de preços dos medicamentos pioneiros de marca ${ }^{48}$ em cada período, $p f_{i t}$, é explicado pelos seguintes variáveis: tempo de entrada em meses do medicamento genérico no mercado do medicamento de marca após a entrada em vigor da lei de genéricos, lagentryit; número de genéricos de cada

\footnotetext{
${ }^{46}$ Somente os introduzidos no país após 1997 tiveram o direito de patentes respeitados.

${ }^{47}$ Por se tratar de valores mensais distanciados de 6 meses se optou por não estimar o modelo em diferenças conforme Caves et alii (1991), pois isso poderia potencializar os efeitos sazonais.

${ }^{48}$ Utilizou-se os preços da ABCFARMA por sua disponibilidade.
} 
medicamento referência existentes em cada período, ngeneit; ${ }^{49}$ idade do medicamento de marca no mercado, idade; idade do medicamento ao quadrado, idade2; dummies de tempo para captar efeitos de variação de custo de produção e efeitos macroeconômicos, tais como variação cambial e variação da inflação, $\mu_{t}$; e resíduo aleatório, que por hipótese deve atender às restrições usuais de ortogonalidade, $\varepsilon_{i t}$.

Os resultados das estimativas com diferentes especificações de $\mathrm{MEF}^{50}$ com as variáveis em nível e seus logaritmos naturais são mostrados na tabela 4. O efeito fixo é considerado por medicamento para levar em conta as diferenças entre cada um destes bens e as suas respectivas parcelas de custo marginal, supostos constantes no tempo. Nas duas primeiras colunas têm-se respectivamente o MEF com controle de dummies de tempo, yt, e o MEF-IV que utiliza uma tendência elevada ao quadrado, trend2, como instrumento para a variação do número de produtores de genéricos no mercado. Nas duas últimas colunas são apresentados os mesmos modelos estimados com os logaritmos naturais das variáveis em acordo com a discussão apresentada na seção anterior.

Tabela 4 - Modelos lineares estimados com as variáveis em nível

\begin{tabular}{|c|c|c|c|c|}
\hline & $\begin{array}{l}\text { MEF i(medicam) } \\
\text { var. em nível }\end{array}$ & $\begin{array}{l}\text { MEF/VI i(medicam) } \\
\text { var. em nível }\end{array}$ & $\begin{array}{l}\text { MEF i(medicam) } \\
\text { var. em logaritmo }\end{array}$ & $\begin{array}{l}\text { MEF/VI i(medicam) } \\
\text { var. em logaritmo }\end{array}$ \\
\hline lagentry & $-0.021(-1.90)$ & $-0.0122(-1.14)$ & $-0.0009(-1.88)$ & $-0.0008(-1.55)$ \\
\hline Ngene & $0.008(0.31)$ & $-0.5096(-6.94)^{*}$ & $-0.0010(-1.26)$ & $-0.0096(-6.65)^{*}$ \\
\hline Idade & $-1.407(-5.82)^{*}$ & Dropped & $-0.0710(-3.40)^{*}$ & $-0.1015(-5.30)^{*}$ \\
\hline idade2 & $0.018(4.69)^{*}$ & $0.0147(4.36)^{*}$ & - & - \\
\hline yt1 & $-2.533(-5.63)^{*}$ & $-2.528(-5.62)^{*}$ & $-0.1098(-11.16)^{*}$ & $-0.1095(-10.62)^{*}$ \\
\hline yt2 & dropped & Dropped & dropped & dropped \\
\hline yt3 & $0.164(0.60)$ & $0.7072(1.24)$ & $-0.0184(-1.77)$ & $0.0081(0.90)$ \\
\hline yt4 & $0.094(0.37)$ & 0.7109 (1.51) & $-0.0154(-1.48)$ & $0.0112(1.25)$ \\
\hline yt5 & $0.117(0.58)$ & $1.0346(2.79)^{*}$ & $-0.0460(-3.78)^{*}$ & $0.0120(1.30)$ \\
\hline yt6 & $-1.408(-5.52)^{*}$ & $-0.124(-0.40)$ & $-0.1251(-9.62) *$ & $-0.05258(-5.18)^{*}$ \\
\hline yt7 & dropped & Dropped & $-0.0757(-5.36)^{*}$ & dropped \\
\hline yt8 & $-0.617(-1.92)$ & $-0.624(-1.94)$ & $-0.1073(-7.61)^{*}$ & $-0.0317(-3.11)^{*}$ \\
\hline cont. & $35.72(14.03)^{*}$ & $17.97(20.69)^{*}$ & $2.7305(55.38)^{*}$ & $2.7952(59.18)^{*}$ \\
\hline n.obs. & 1218 & 1218 & 1218 & 1218 \\
\hline
\end{tabular}

Os valores entre parênteses são os resultados das estatísticas de teste.

Nos MEFs a variável que indica o aumento do número de medicamentos genéricos em cada período, ngene, não se mostrou estatisticamente significativa, sugerindo que o aumento da concorrência do genérico não teria alterado a decisão de preços do produtor do medicamento pioneiro. Entretanto, estes modelos não consideram a possibilidade do número de medicamentos genéricos ser endógeno em relação ao preço do seu referência, pois numa economia em que todas as pessoas têm acesso aos medicamentos, a única possibilidade para um produtor aumentar seu faturamento é através do aumento de seu preço. Este comportamento, todavia, sinaliza grandes margens de lucro aos potenciais entrantes produtores de medicamentos genéricos, implicando num duplo sentido de causalidade entre a decisão de preço do medicamento de referência e a decisão de entrada dos genéricos.

Os MEF-IVs, tabela 4, levam em consideração o potencial problema de endogeneidade, entre preço do medicamento referência e número de genéricos, através do uso de variável instrumental. A variável

\footnotetext{
${ }^{49} \mathrm{O}$ número de medicamentos genéricos é maior que o número de seus produtores, pois um mesmo medicamento referência pode ter mais de uma apresentação de genérico.

${ }^{50}$ Para todos eles o teste de especificação de Hausman rejeita o uso de modelo de efeito aleatório.
} 
instrumentada, que descreve o número de genéricos ao longo do tempo, mostrou-se estatisticamente significativa e com sinal negativo, sugerindo que o aumento do número de genéricos de um mesmo medicamento de referência no mercado tendeu a reduzir os preços destes últimos no período analisado.

$\mathrm{O}$ uso da tendência ao quadrado como instrumento do número de genéricos no mercado se justifica pela idéia de que a entrada de novos produtores de genéricos é crescente e finita. Além disso, o medicamento genérico tem um ciclo de vida menor do que o do seu medicamento de referência. Assim, tal instrumento, que apresenta razoável grau de associação linear, 0,48, com a variável instrumentada, parece bastante adequado, uma vez que se espera não haver correlação deste instrumento com o resíduo porque foram introduzidas dummies de tempo no modelo que devem captar possíveis efeitos de tendência.

A transformação logarítmica das variáveis não altera de maneira significativa os resultados obtidos, basicamente os sinais e nível de significância dos coeficientes estimados são os mesmos, a menos dos coeficientes associados com as dummies de tempo que se tornam significativos com sinal negativo, evidenciando a queda dos preços dos medicamentos referência no período estimado.

A variável idade no segundo modelo é eliminada pelo programa devido à colinearidade com as variáveis binárias. Mas em todos os modelos se verifica que os coeficientes associados às variáveis que descrevem a idade e a idade ao quadrado dos medicamentos - polinômio que deve captar o ciclo de vida do produto - são estatisticamente significantes e possuem respectivamente sinais negativos e positivos. No caso da transformação logarítmica a idade ao quadrado é eliminada por ser combinação linear do logaritmo da idade. Esses resultados fornecem mais evidências de que o ciclo de vida do medicamento "médio" no Brasil, avaliado pelos medicamentos disponíveis na amostra de dados deste estudo, não têm o padrão esperado pela teoria do ciclo de vida tradicional.

Os resultados dos modelos estimados, mostrados na tabela 4 também indicam que os preços dos medicamentos de marca no Brasil não responderam às defasagens de entradas dos medicamentos genéricos em seus mercados. A variável que mede a demora da entrada dos medicamentos genéricos no mercado, lagentry, apresenta coeficiente negativo e estatisticamente não significativo em todos os modelos estimados. Este resultado era esperado e deve refletir dois fenômenos distintos além do curto período da existência de genérico no mercado brasileiro. O primeiro é que a entrada do genérico se tornou possível - por lei, a partir de 1999 - ${ }^{51}$ simultaneamente em praticamente todos os mercados específicos de medicamentos pioneiros produzidos no país. Além disso, como a lei de patentes passou a vigorar somente em 1997, não existe ainda, ${ }^{52}$ no Brasil, medicamentos com patentes a expirar num curto período de tempo, caracterizando uma situação bastante diferenciada da estudada por Caves et alii (1991) no mercado americano. O segundo fenômeno se refere ao fato de que grande parcela dos medicamentos genéricos que entrou no mercado brasileiro ser composta pelos já comercializados medicamentos similares, que se submeteram ao teste de bioequivalência e tiveram sua produção continuada. Assim, como existia um prazo final - até setembro de 2001 - para que os "falsos genéricos" mudassem de nome, muitos deles efetivamente entraram nesta data como genéricos, ${ }^{53}$ de modo que a lógica econômica que levou a decisão de entrada do genérico foi a de ganho de escala.

Com relação aos efeitos da regulação do setor entre 2001 e 2002 pode-se dizer que todos os medicamentos pioneiros de marca estudados estiveram submetidos a esta política. Inclusive este fato pode explicar a tendência de queda nos seus preços verificada no modelo, captada pelos coeficientes negativos e significativos das dummies de tempo referentes ao final da amostra. Isto, entretanto, não interfere no resultado obtido de que quanto maior o número de genéricos no mercado de um medicamento referência, menor o preço do medicamento de referência, resultado captado pela significância e

\footnotetext{
${ }^{51} \mathrm{Na}$ prática o governo só teve habilidade de prover os testes de bioequivalência e controles somente a partir de 2000, quando os medicamentos genéricos efetivamente entraram no mercado brasileiro.

${ }^{52}$ Este trabalho foi finalizado em 2003.

${ }^{53}$ Embora uma grande parte dos falsos genéricos não tenha conseguido autorização para se tornarem genéricos.
} 
pelo sinal negativo do coeficiente da variável que descreve o número de genéricos em cada mercado a cada período.

\section{CONCLUSÕES}

Os resultados deste artigo apontam para um efeito negativo sobre os preços dos medicamentos pioneiros de marca que se tornaram referência em resposta à entrada dos genéricos em seus respectivos mercados. Além disso, sugerem que os preços dos medicamentos de referência são sensíveis negativamente ao aumento do número de genéricos em seus mercados. Estas tendências são particularmente interessantes do ponto de vista do bem-estar da sociedade brasileira, pois se os preços dos medicamentos de marca são reduzidos como resposta ao aumento da concorrência, então, tende a ocorrer uma queda no custo de tratamento de males ou doenças, e conseqüentemente, uma melhora para os consumidores, pois o medicamento genérico, pela própria lógica de concorrência, entra com preço menor que o do seu medicamento de referência.

Para avaliar se houve melhora de bem estar, entretanto, torna-se necessário verificar, em trabalhos futuros, se houve aumento da quantidade consumida destes bens. Tal análise deve investigar se a soma das quantidades dos medicamentos de referência e genéricos após a entrada destes últimos no mercado é maior, igual ou menor que a quantidade total consumida do medicamento de referência antes da concorrência.

Os resultados deste artigo sugerem indiretamente que houve uma mudança significativa no comportamento dos produtores dos medicamentos pioneiros frente aos medicamentos genéricos em relação à maneira como se comportavam diante da concorrência oferecida pelos medicamentos similares. Esta mudança pode ter ocorrido por dois motivos: o governo, com a institucionalização do medicamento genérico através da exigência do teste de bioequivalência aliado à criação da ANVISA sinalizou para o mercado que o medicamento genérico é um produto de boa qualidade; e os grandes gastos do governo com publicidade sobre os medicamentos genéricos atuaram de maneira semelhante aos grandes gastos com propaganda realizados pelos grandes laboratórios farmacêuticos que criam fidelidade aos produtos.

Em ambos os casos houve uma melhora de informação sobre a qualidade do medicamento genérico. Entretanto, vale notar que no Brasil um medicamento genérico passa uma única vez pelo teste de bioequivalência, quando necessita da autorização para entrar no mercado. Levando em conta a experiência americana, seria interessante que o controle de qualidade institucional ocorresse periodicamente, para evitar problemas de perda de qualidade do medicamento genérico e, conseqüentemente, problemas de perda de credibilidade por parte dos consumidores, que poderiam inviabilizar a política de genéricos no futuro.

\section{Referências Bibliográficas}

Achilladelis, B. \& Antonakis, N. (2001). The Dinamics of Tecnological Innovation: Case of the Pharmaceutical Industry., volume 30. Research Policy.

Aghion, P. \& Howitt, P. (1992). A model of growth through creative destruction. Econometrica, 60(2).

Bermudez, J. A. Z., Epsztejn, R., Oliveira, M., \& Hanseclever, L. (2000). O acordo TRIPS da OMC e a Proteção Patentária no Brasil: Mudanças recentes e implicações para a produção local e o acesso da população aos medicamentos. Technical report, Fundação Oswaldo Cruz, Centro Colaborador da OPAS/OMS, Rio de Janeiro.

Bernardo, J. B. \& Romano, R. (2001). Padrões de regulação de preços do mercado de medicamentos: Experiência Brasileira dos anos 90 e novos arranjos institucionais. Technical report, Org. B.Negri e G. Di Giovanni, Instituto de Economia-UNICAMP, Brasil Radiografia da Saúde. 
Caves, R. E., Whisthon, M. D., \& Hurwitz, M. A. (1991). Patent Expiration, Entry, and Competition in the U.S. Pharmaceutical industry. Brookings Papers: Microeconomics.

Danzon, P. M., Epstein, A., \& Nicholson, S. (2004). Mergers and acquisitions in the pharmaceutical and biotech industries. Technical Report 10536, National Bureau of Economic Research.

Fiúza, E. P. S. \& Lisboa, M. B. (2001). Bens credenciais e poder de mercado: Um estudo econométrico da indústria farmacêutica Brasileira. Technical report, Texto para Discussão do IPEA, Rio de Janeiro.

Frank, R. G. \& Salkever, D. S. (1991). Pricing, patent loss and market for pharmaceuticals. Technical Report 3803, National Bureau of Economic Research.

Grabowski, H. \& Vernon, J. (1992). Brand loyalty, entry and price competition in pharmaceutical after the 1984. The Journal of Law and Economics, XXXV.

Griliches, Z. (1961). The Price Statistics of the Federal Government, volume 73, chapter Hedonic Price Indexes for Automobiles: An Econometric Analysis of Quality Change, pages 137-196. General Series.

Griliches, Z. (1989). Patents: Recent trends and puzzles. Technical Report 2922, National Bureau of Economic Research, Inc.

Griliches, Z. \& Cockburn, I. (1993). Generics and new goods in pharmaceutical price index. Technical Report 4272, National Bureau of Economic Research.

Grossman, G. M. \& Lai, E. L. C. (2002). International protection of intellectual property. Technical Report 8704, National Bureau of Economic Research.

Hurwitz, M. A. \& Caves, R. E. (1988). Persuasion or information? promotion and the shares of brand name and generic pharmaceuticals. The Journal of Law and Economics, XXXI.

Kamien, M. K. \& Zang, I. (1999). Virtual patent extension by cannibalization. Southern Economic Journal, $66: 117-131$.

Kremer, M. (1994). Patents buy-out: A mechanism for encoraging innovation. Technical Report 6304, National Bureau of Economic Research.

Lanjouw, J. O. (1998). The introduction of pharmaceutical product patents in India: Heartless Exploitation of the Poor and Suffering? Technical Report 6366, National Bureau of Economic Research.

Lanjouw, J. O. \& Cockburn, I. (2000). Do patentes matter?: Empirical evidence after gatt. Technical Report 7495, National Bureau of Economic Research.

Lee, M. J. (2005). Micro-Econometrics for Policy, Program, and Treatment Effects - Advanced Texts in Econometrics. Oxford Press.

Lichtenberg, F. R. (2001). The benefits and costs of newer drugs: Evidence from the 1996 medical expenditure panel survey. Technical Report 8147, National Bureau of Economic Research.

McClellan, M. (1995). Uncertainty, health-care technologies, and health-care choices. AEA Papers and Proceedings, 85(2).

Nishijima, M. (2003). Análise econômica dos medicamentos genéricos no Brasil. Technical report, Tese de Doutorado IPE-USP, São Paulo.

Pavcnik, N. (2000). Trade liberalization, exit and productivity improvements: Evidence from Chilean plants. Technical Report 7852, National Bureau of Economic Research. 
Rosenthal, R. W. (1980). A model inwith an increase in the number of sellers leads to a higher price. Econometrica, 48(6).

Smith, P. J. (2002). Patent Rights and Trade: Analysis of Biological Products, Medicinals and Botanicals, and Pharmaceuticals. American Journal of Agricultural Economics, 84(2):495-512.

Watanabe, C., Takayama, M., Nagamatsu, A., Tagami, T., \& Griffy-Brown, C. (2000). 'Technology Spillover as a Complement for High-Level RED Intensity in Pharmaceutical Industry. Technovation. 